\title{
Cloud-based Atlassing
}

\author{
Florian Hruby ${ }^{1}$, Rainer Ressl ${ }^{1}$ and Alexander Wolodtschenko ${ }^{2}$ \\ ${ }^{1}$ National Commission for Knowledge and Use of Biodiversity (CONABIO), Mexico City · \\ florian.hruby@conabio.gob.mx \\ ${ }^{2}$ Dresden University of Technology, Germany
}

Full paper double blind review

\begin{abstract}
The Internet has evolved into a powerful tool for cartography, highly useful both from the mapmaker's and the user's point of view. With the recent emergence of cloud computing, these benefits are taken even a step further. Using the example of atlases, this article discusses theoretical and practical opportunities of cloud-based cartography. Two broad topics are dealt with. The first, addressed in sections 1 and 2, concerns how cartography gradually evolved into the cloud, and how cloud computing can provide solutions for the challenges faced by geospatial sciences. The second topic, covered in chapters 3,4 and 5, addresses issues of atlas cartography: starting with a critical review on current literature, we will argue that the fundamental characteristic of digital atlases is not so much the use of digital maps, but rather the combination of these maps. Based on this assumption we will show, how digital atlases facilitate the synthesis of different maps, and how atlas production can benefit from a cloud-based approach. We shall close the paper with a look at how the cloud can transform future school atlases from products to processes (termed 'atlassing'), executed in a stand-alone mode, or as part of learning management systems.
\end{abstract}

\section{From Analog Mapping to Cloud-based GIS-Cartography}

Analog maps have been the most important medium of cartographic representation for centuries. In the pre-digital era, maps fulfilled at least three functions, i.e. (1) to visualize, (2) to analyze and (3) to store spatial data. This multi-functionality of analog maps started to change with the arrival of the first geographic information systems (GIS) in the 1960s (see figure 1): each GIS comes along with powerful tools for spatial analysis on data stored in a geodatabase. Hence, in a GIS-based workflow, the map's principal task is to visualize the data stored in the database and the results of analysis done on this data. Despite such functional division, early GIS usually organized their components (hardware, software, data) centrally on one computer.

However, with the rise of the Internet, a spatial division has increasingly paralleled this functional division: in a first step, the Internet allowed the publication of GIS-made maps online in a static format. As of the late 1990s, web interfaces designed for GIS also allowed for an interactive map interpretation and map production online, usually building on clientserver architecture. In these "online GIS", the user is presented with a web page that allows a query to be made of a database. The resulting map is then displayed within a web page, and further controls are provided to perform another query" (PETERSON 2014). 
While the online GIS approach uses data centers mainly for data storage and retrieval, cloud computing capitalizes their processing power as well: "Cloud computing is a model for enabling ubiquitous, convenient, on-demand network access to a shared pool of configurable computing resources (e.g. networks, servers, storage, applications, and services) that can be rapidly provisioned and released with minimal management effort or service provider interaction" (MELL \& GRANCE 2011). Due to the high processing power, the computationally expensive analysis processes of a GIS can also be migrated into the cloud. And although the analyses functions of current cloud-based GIS solutions (e.g. ESRI's ArcGIS Online) are still limited, it is foreseeable that future GIS won't be necessarily installed on a local drive, but accessed as a service through the browser.

Compared with the aforementioned server-client based online GIS, a cloud-based GIS can deliver significant benefits, not only in processing power enhancement, but also in cost savings: "The advantage is more flexibility in server environments and greater data security. Cloud-based solutions are also usually less expensive" (PETERSON 2014).

To sum up this introduction: cloud-based GIS can be understood in consequence of the functional division that started with the first GIS in the 1960s. In current GIS workflows, data, software, and/or maps are often hosted in remote data centers connected through the Internet (see figure 1). Hence, in many cases we not only work on data, which is not stored on our local computer, but we also don't know where the data and the software to analyze and visualize this data are located. To express this lack of knowledge metaphorically, and to describe the remotely networked architecture, the term 'cloud' is used for the data centers we rely on. Although the origin of the term itself seems to be unclear yet, it has become increasingly popular, as "it captures a historic shift in the IT industry as more computer memory, processing power, and apps are hosted in remote data centers, or the 'cloud' " (REGALADO 2011). We will present this concept in further detail in the following chapter.

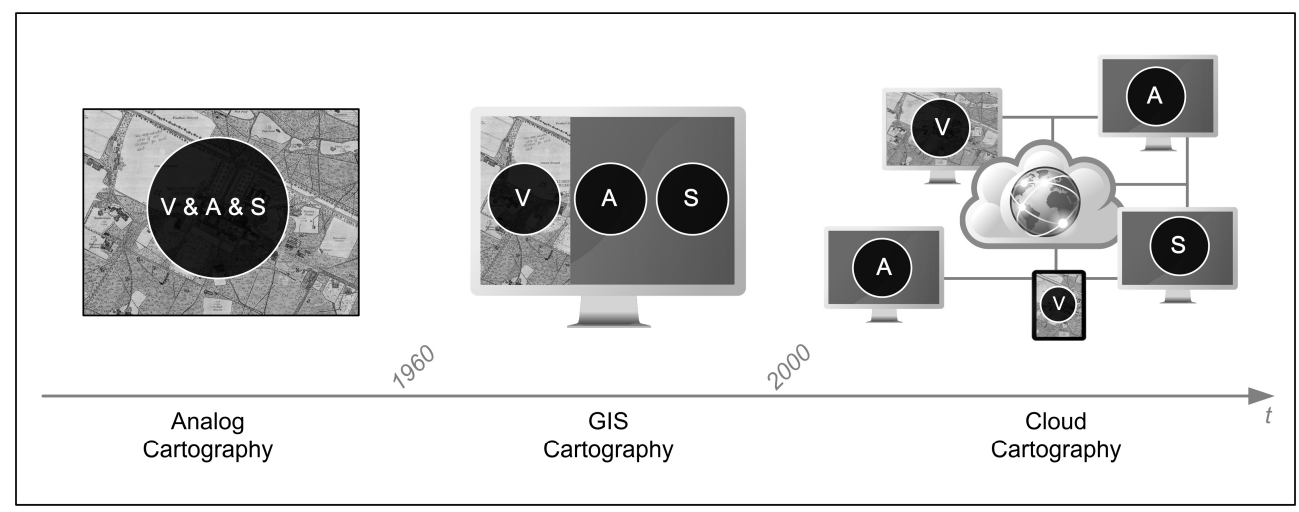

Fig. 1: From analog to cloud-cartography - the basic tasks of analog maps [(visualize (V), analyse (A), store (S)] and their transformation into the cloud 


\section{Cloud Computing \& Cloud Cartography}

As we have outlined above, cloud computing and cloud-based cartography can be understood in succession of earlier technological developments. To put it with ARMBRUST et al. (2009) it is "an old idea whose time has (finally) come". The basic principle is to deliver on-demand computing services through the Internet. Depending on the kind of service provided, the following threefold division, the so called SPI service model, is usually made (cf. HAMDAQA \& TAHVILDARI 2012, MELL \& GRANCE 2011):

- Infrastructure-as-a-Service (IaaS): delivering infrastructure like hardware, storage (e.g. for geospatial data) or system software.

- Platform-as-a-Service (PaaS): delivering platform services like Application Programming Interfaces (APIs) that allow the consumer to develop applications onto a cloud infrastructure (e.g. ESRI's ArcGIS for JavaScript API, or the Google Earth API).

- Software-as-a-Service (SaaS): delivering and using software applications (e.g. ArcGISOnline), usually through a web browser.

Aside from these three service models, cloud computing shows five characteristics, which "differentiate cloud computing from other distributed computing paradigms, such as grid computing": (1) on-demand self-service, (2) broad network access, (3) resource pooling, (4) rapid elasticity, and (5) measured service (MELL \& GRANCE 2011). These characteristics, on the one hand, bring economic benefits for customers. For instance, users do not need to buy hardware or software nor care about update and maintenance work, but rather lease the necessary capacities on a pay-for-use basis. On the other hand, cloud computing benefits from the multiplied processing power of data centers. As a result, "several important classes of existing applications will become even more compelling with Cloud Computing and contribute further to its momentum" (ARMBRUST et al. 2010), e.g.:

- Mobile real-time applications: highly available services, often resulting from a combination of different data sources (e.g. mashups) and able to process real-time data, are particularly suited for a cloud-based delivery model.

- Compute-extensive application: computationally intensive processes like animations, $3 \mathrm{D}$ visualizations and big data analysis (e.g. of Earth observation data) profit by highperformance distributed computing resources.

- User analytics: cloud computing helps to realize data- and compute-intensive customer analysis, in order to better understand patterns of use.

As the above examples already indicate, cloud computing can also be useful for geoscientific concerns. Moreover, the cloud provides a solution to the information technology (IT) challenges faced by geospatial science, which have been defined by YANG et al. (2011): (1) data intensity (e.g. petabytes of daily captured satellite imagery data), (2) computing intensity (e.g. to analyze and model Earth observation data), (3) concurrent intensity (e.g. to enable massive concurrent user access to data and data visualization), and (4) spatiotemporal intensity (e.g. to work on spatiotemporally high resolution geodata).

The aforementioned arguments refer to general advantages of cloud computing for geodata analysis. Given this, we can narrow the focus further on typical issues of cartography, i.e. on the cloud's potential for cartographic visualization. Already, a large part of the maps available online are stored in the cloud, mostly tile-based (cf. SAMPLE \& IOUP 2010). Consequently we may consider each map in terms of the cloud-computing paradigm as visuali- 
zation-as-a-service (VaaS). Hence, to make full use of these technological developments, modern cartographers need to have both practical and theoretical knowledge on how to use the cloud for mapmaking. "Otherwise, a theoretical cartographer cannot make a map and a practical cartographer does not know what it means" (PETERSON 2013). In addition to the term 'cloud computing', we propose the term 'cloud cartography' in order to label the potential of cartographic visualizations based on the cloud. Extending the SPI model by a VaaS layer is considered to be a defining characteristic of cloud cartography (see figure 2).

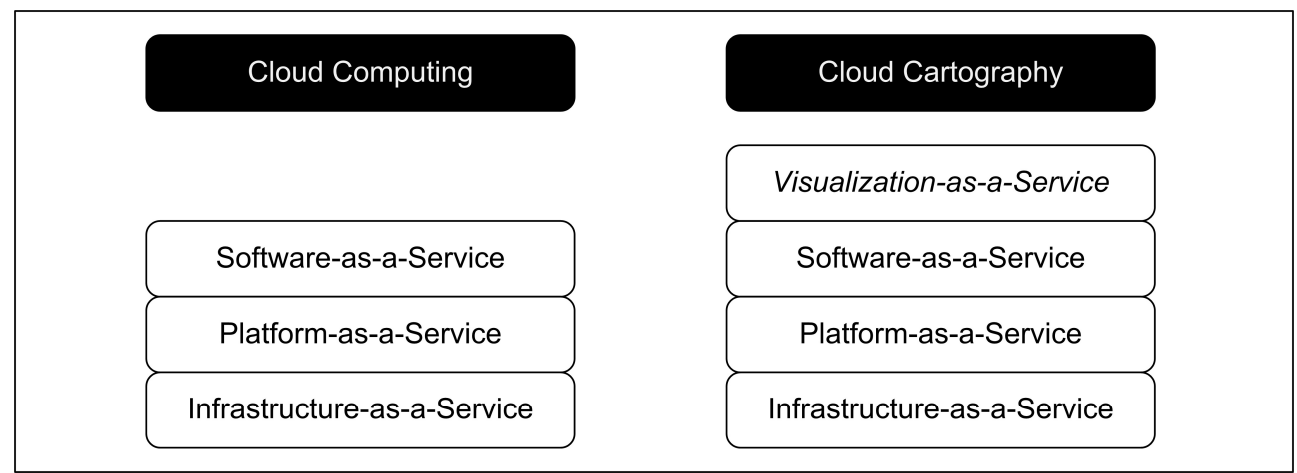

Fig. 2: Service models of cloud computing and cloud cartography

To summarize this chapter, we can state that many of the current geodata processing issues can benefit from high-performance cloud computing. In fact, cloud computing and geospatial sciences complement and advance each other: "[C]loud computing not only could help geospatial sciences, but can be optimized with geospatial principles to best utilize available distributed resources" (YANG et al. 2011). In order to further develop the concept of cloud cartography, and to illustrate its potential for cartographic visualization, the following sections will be devoted to atlases, which, due to their complexity, can give a suitable example for this purpose.

\section{Atlas and Atlas Cartography}

In a nutshell we can define atlases as “... intentional combinations of maps or data sets, structured in such a way that specific objectives are reached" (KRAAK \& ORMELING 2009). Such an intentional combination generates a structure for each atlas, which is appropriately described as "geographic narrative" (ibid.). Possible narrative structures are for example:

- Spatial structures: for instance, a school atlas may start with maps on a national level, followed by continental maps and concluding with small scaled world maps.

- Thematic structures: a national atlas may start with topographic and thematic maps on general characteristics of a country, like climate, geology, soil, etc., followed by more specific topics, e.g. agricultural land, which can be understood in consequence of the former maps.

- Temporal structure: a history atlas, for example, presenting maps on a particular region's history in a chronological order. 
- Comparative/presentational structure: order and arrangement of individual maps can shape the aforementioned narrative structures. For instance, we can combine two or more maps in a manner that facilitates spatial, thematic and/or temporal comparison, like a double page in a paper atlas where each sheet shows a map of the same region at a different time (e.g. 1950 vs. 2000).

- Common/'Corporate' design: in order to strengthen geographic narratives, an atlas usually builds on a common map language and presentation, resulting in a consistent atlas design. For example, the same types of objects will be represented by the same map symbols throughout the whole atlas, so that the user does not need to relearn symbolization rules for each map.

According to the chosen narrative structure(s), atlases can be subdivided in different ways, both regarding their content (e.g. geography vs. history, or topographic vs. thematic atlases, etc.) and in respect to their particular purpose (e.g. education, planning, reference, etc.), (cf. KRAAK \& ORMELING 2009, VOZENILEK 2015).

Moreover, with the emergence of digital cartography, a division in analog and digital atlas has been proposed repeatedly (cf. DA SILVA RAMOS \& CARTWRIGHT 2006). Taking a closer look at this differentiation, it is striking to note that most of the common arguments fall short. Attributes typically assigned to digital atlas are: dynamic, interactive, multimedia, zoomable, among others. But these features are differences between digital and analog maps in general. Consequently, given that an atlas is more than the sum of its maps, attributes like dynamic or interactive are not suitable distinguishing features to describe requirements and potential of digital atlases. As mentioned initially, a defining characteristic of an atlas is not so much the usage of maps, rather the combination of maps. However, scientific literature on atlas cartography generally does not address the issue of whether digital atlases can offer fundamentally different combinations of maps than analog atlases do. The following chapter shall consider this question in more detail.

\section{Digital vs. Analog Atlases}

The basic task of an atlas is to support synthesis and comparison of map contents. Aside from map order within the whole atlas, both analog and digital atlases try to facilitate comparison between maps by offering two or more maps at one glance. As a function of space available, several maps can be directly compared using various techniques, e.g. pairwise juxtaposition or "small multiples" in terms of TUFTE (1990). Basically, this applies for both paper and onscreen atlases. However, in an analog atlas, the possible order of maps is limited in a one-dimensional way:

Let us imagine a map $\mathrm{M}_{\mathrm{A}}$, which occupies the left half of a double page of an analog atlas. $\mathrm{M}_{\mathrm{A}}$ shows a particular place (P1), at a particular point of time (T1) on a particular subject (S1). Like most common maps, $\mathrm{M}_{\mathrm{A}}$ is spatially, temporally and thematically referenced. Accordingly we can describe $\mathrm{M}_{\mathrm{A}}$ by a combination of: $\mathrm{P} 1 / \mathrm{T} 1 / \mathrm{S} 1$.

Let us further imagine that $M_{A}$ is the basis of reference for a second, comparative map $M_{B}$, which is shown on the right half of the same double page. To allow for a meaningful comparison, $\mathrm{M}_{\mathrm{B}}$ can visualize (see also figure 3 ): 
- firstly, the same place (P1) at the same time (T1), but on a different subject (S2), resulting in $\mathrm{M}_{\mathrm{B}-1}$ : $\mathrm{P} 1 / \mathrm{T} 1 / \mathrm{S} 2$. In this case the comparison between $\mathrm{M}_{\mathrm{A}}$ and $\mathrm{M}_{\mathrm{B}-1}$ would have a thematic narrative structure.

- secondly, the same place (P1) at a different point of time (T2), on the same subject (S1): $\mathrm{P} 1 / \mathrm{T} 2 / \mathrm{S} 1$. In this scenario we could compare $\mathrm{M}_{\mathrm{A}}$ with $\mathrm{M}_{\mathrm{B}-2}$ on the basis of a temporal narrative structure.

- thirdly, a different place (P2) at the same time (T1), on the same subject (S1), so that $\mathrm{M}_{\mathrm{B}-3}$ would be a combination of: P2/T1/S1. A spatially structured comparison would be the result of this case.

Since one of these three narrative structures will always dominate a comparison between two analog atlas maps, we can speak of a one-dimensional (i.e. horizontal) comparison. Digital atlases allow adding a second (i.e. vertical) dimension to the horizontal structure mentioned. To continue with the exemplary atlas page sketched above, an additional vertical dimension enables users to interactively switch between the three comparative maps $\mathrm{M}_{\mathrm{B}-1,}, \mathrm{M}_{\mathrm{B}-2}$ and $\mathrm{M}_{\mathrm{B}-3}$, while the basis of reference $\mathrm{M}_{\mathrm{A}}$ would remain permanently visible, e.g. on the left side of a digital atlas interface. Likewise, modifications could be realized within the same narrative structure, for example by changing the comparative map $\mathrm{M}_{\mathrm{B}}$ iteratively from $\mathrm{P} 1 / \mathrm{T} 2 / \mathrm{S} 1$ to $\mathrm{P} 1 / \mathrm{T} 3 / \mathrm{S} 1, \mathrm{P} 1 / \mathrm{T} 4 / \mathrm{S} 1$, etc. (e.g. by means of animation techniques). Translating such a vertical structure into the horizontal structure of an analog atlas would be possible only to a limited extent, and with considerably more effort (see figure 3 ).

Moreover, the digital version of our exemplary atlas permits to exchange the map of reference for the comparative map, and vice versa: the comparative map $M_{B}$ in figure 3 can become the map of reference, when we start to modify $M_{A}$, while $M_{B}$ will not change. Thinking ahead with this idea, we could modify $\mathrm{M}_{\mathrm{A}}$ and $\mathrm{M}_{\mathrm{B}}$ even in a parallel fashion, for instance by synchronizing the scale of both maps dynamically.

The differences between analog and digital atlases that we have seen so far concern the change of dynamic maps within a static atlas interface. As a matter of course, digital atlases also allow for an implementation of dynamic interfaces, which give way to new forms of map comparison and analysis. Figure 4 illustrates these possibilities by means of two examples: in both cases, the maps to be compared are not placed side by side, as it needs to be done in a horizontally structured analog atlas, but rather superimposed, so that one map (e.g. $\mathrm{M}_{\mathrm{A}}$ ) lies on top of another (e.g. $\mathrm{M}_{\mathrm{B}}$ ), each of them representing the same place (e.g. P1). Various techniques can be used to contrast both maps. In the case of interface A, a vertical slider allows to define, which part of each map will be visible for the user, following the idea that swiping this slider dynamically uncovers differences more easily than a comparison of two static maps would do. In the case of interface B, the underlying map is visible through a spyhole in the overhead map, which can be moved by the user. 


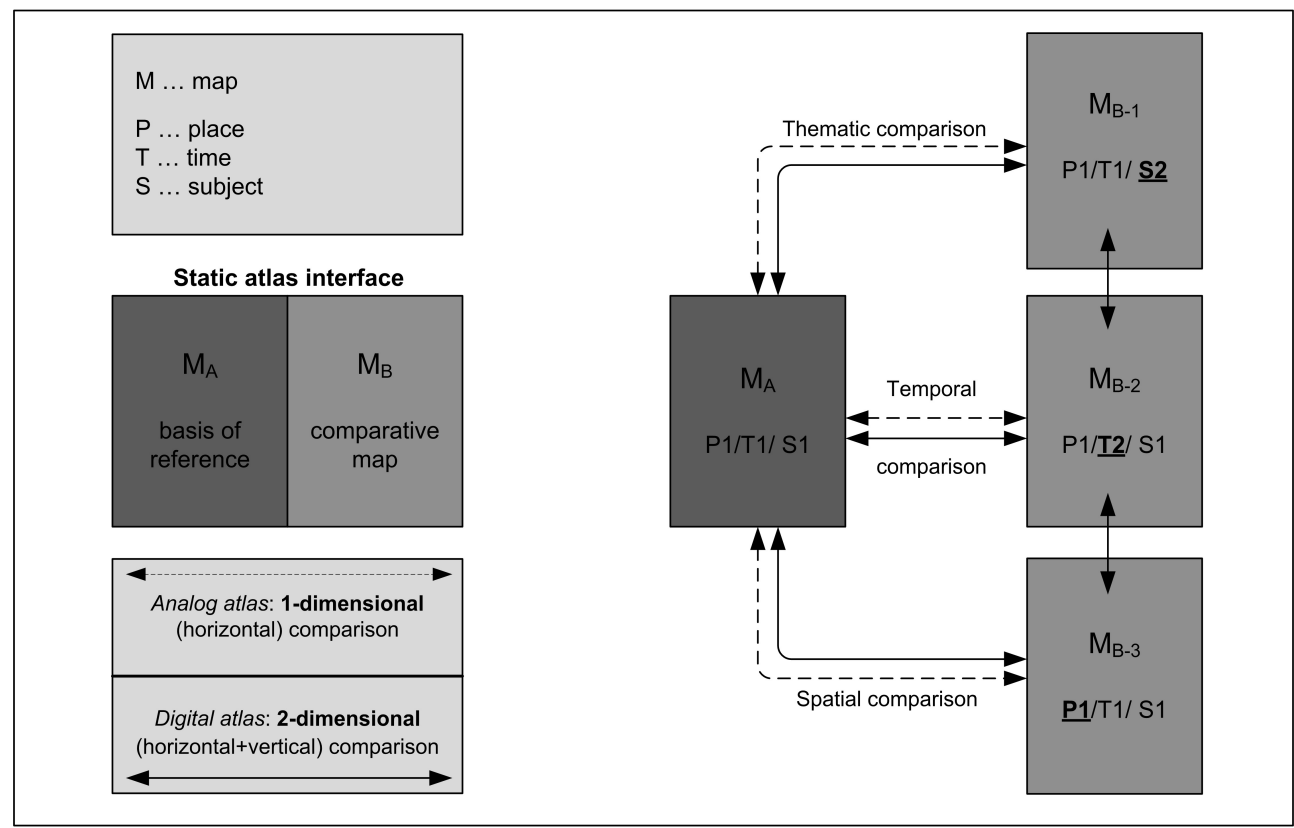

Fig. 3: 1-dimesional vs. 2-dimensional narrative structures in analog and digital atlases

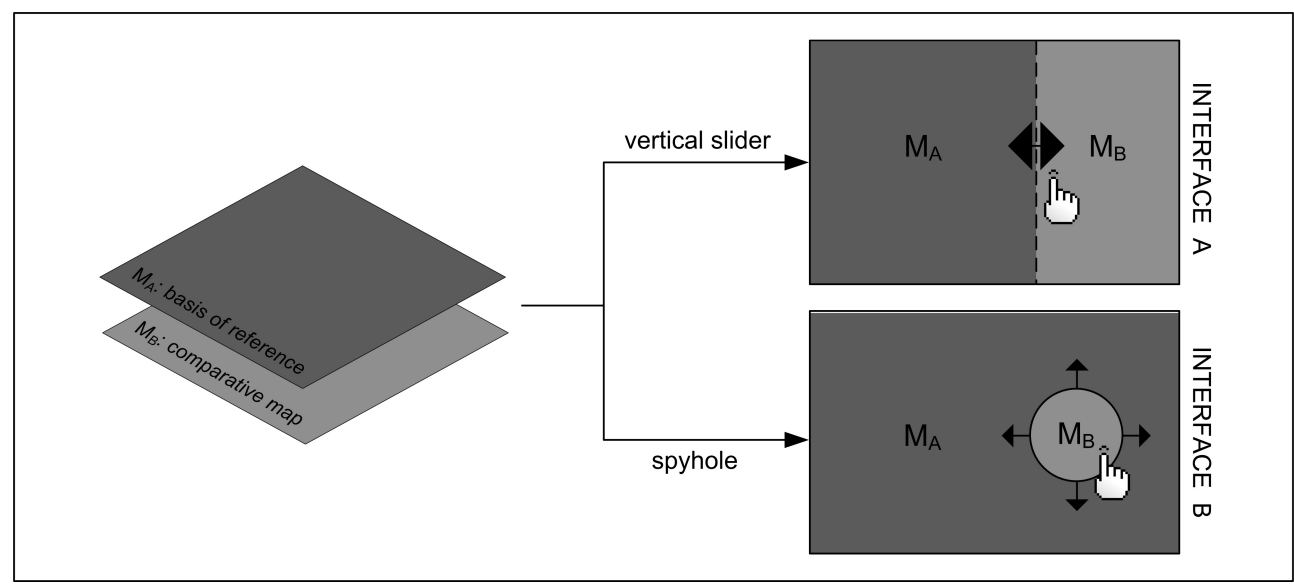

Fig. 4: Examples of map comparisons in dynamic atlas interfaces

In this section, we focused on special features of digital atlases for the comparison of two or more maps within static or dynamic atlas interfaces. These features are, strictly speaking, nothing that cannot be done without cloud cartography, but the cloud's benefits on performance and costs make their implementation significantly easier. As we shall see in the next section, the cloud not only facilitates the synthesis of different maps in a digital atlas, but also opens up new perspectives on how atlases can be produced. 


\section{$5 \quad$ School Atlassing}

From the user's point of view, each analog atlas is a completed product whose content cannot be modified significantly (ORMELING 1996). The same applies for early digital atlases delivered offline on CD or DVD, while later products can be complemented with online data as well, at least to a certain extent. Since the emergence of cloud computing, the number of themes and maps that can be combined into an atlas is, at least in principle, infinite. Users can not only compile existing maps according to their needs, but also produce their own atlas from scratch, i.e.: (1) collect geodata, (2) edit and analyze the data in a GIS, (3) make maps from the data, and (4) compose a digital atlas from these maps. Here, we don't just refer to simple editing features offered by current geobrowsers (e.g. Google Earth), but rather think about more complex analysis and visualization tools of a GIS, which can be accessed cloud-based as software services.

School cartography may give an example of how cloud-based atlases can be used for teaching purposes: usually, analog school atlases are designed for a particular level of education or a particular subject area (e.g. historical atlases). Moreover, their contents are limited as a function of weight and costs. Based on the cloud, this range of use in educational institutions can be expanded significantly. For example, teachers can compile age- and subjectappropriate atlases from a pool of privately or publicly provided maps that can be assembled and compared by means of different predefined interfaces (e.g. figure 4). Corporate design issues can be solved via Cascading Style Sheets (CSS) to assure a consistent appearance of interface, map symbols, widgets, etc. within the whole atlas. Moreover, not just teachers, but also students can get involved into this process of atlas composition, e.g. by presenting a particular topic with the aid of a self-compiled atlas.

Developing this approach a step further - and the cloud supports this step significantly - an atlas no longer needs to be understood as a product consisting of a combination of already existing maps, but rather as a process including data acquisition and mapmaking as well. Hence, learning targets of atlas-aided teaching can include - aside from traditional map reading tasks - basic principles of map making as well, helping students to develop cartographic core competencies.

Open source GIS and cloud-based GIS allow running through the steps of atlas compilation at a low cost level (only computer hardware and internet access is needed). Consequently, students can work actively on the whole process of atlas production - a process which we choose to label with the term 'atlassing' (coined by WOLODTSCHENKO 2012). For the abovementioned benefits of cloud computing, this atlassing process will be necessarily a cloud-based one.

Finally, to better understand the potential of atlassing for knowledge transfer, it should be considered that maps and atlases are only one of many teaching tools that can be transferred into the cloud. In such a scenario, an atlas won't just be a combination of maps, but rather a combination of different learning materials, incl. text, images and other multimedia content resulting in a collaborative learning environment. First examples have already been published online, e.g. the WileyPLUS Learning Space (MCLAREN 2014). 


\section{Conclusion}

In this paper, we tried to show that cloud-based mapping is not to be understood as a completely new paradigm of cartography, but rather in consequence of earlier technological developments in the IT and GIS sector. These advancements imply more processing power at lower costs, which is also of interest for cartographic concerns, as we have illustrated for the case of atlas cartography. Using the example of atlases, it was demonstrated that not only technical, but also theoretical shortcomings hinder enhancement of cartographic visualization. Lacking understanding of how maps can be combined into a digital atlas interface is a consequence of this situation. This issue will concern spatial cognition research as well, since we not only need to know, how maps can be combined, but also how they should be combined to facilitate interpretation and synthesis. Here, cloud-based cartography can trigger theoretical, empirical and practical research, which will benefit mapmaking in general, and the understanding of cartographic atlases in particular. Regardless of whether a digital atlas is designed as a stand-alone combination of maps, or rather as an integrated part of learning management systems, we can conclude with PETERSON (2013): "Cloud computing is clearly the future for online mapping".

\section{References}

Armbrust, M., Fox, A., Griffith, R., Joseph, A., Katz, R., Konwinski, A., Lee, G., Patterson, D., Rabkin, A., Zaharia, M. \& Stoica I. (2009), Above the clouds: A Berkeley view of cloud computing. EECS Department, University of California, Berkeley, Technical Report UCB/EECS-2009-28. Retrieved March 30, 2015 from http://www.eecs.berkeley.edu/Pubs/TechRpts/2009/EECS-2009-28.pdf.

Da Silva Ramos, C. \& Cartwright, W. (2006), Atlases from Paper to Digital Medium. In: Stefanakis, E., Peterson, M., Armenakis, C. \& Delis, V. (Eds.), Geographic Hypermedia. Springer, 97-119.

HAMDAQA, M. \& TAHVILDARI, L. (2012), Cloud Computing Uncovered: A Research Landscape. Advances in Computers, 86, 41-85.

KrAAK, M. \& ORMELInG, F. (2009), Cartography: Visualization of Spatial Data. Routledge, $204 \mathrm{p}$.

MCLAREN, B. (2014), What Happens When We Learn Together: A Research-Based Whitepaper on the Power of Collaborative Learning. Wiley \& Sons.

http://issuu.com/wiley_publishing/docs/wpls_whitepaper?e=1085234/10379282

(March 30, 2015).

Mell, P. \& GRAnCE, T. (2011), The NIST Definition of Cloud Computing. NIST Special Publication 800-145.

http://csrc.nist.gov/publications/ nistpubs/800-145/SP800-145.pdf (March 30, 2015).

Ormeling, F. (1996), Functionality of Electronic School Atlases. In: KÖBBEN, B., ORMELING, F. \& TraINOR, T. (Eds.), Proceedings of the Seminars on Electronic Atlases in Prague and The Hague. ICA, 33-39.

Peterson, M. (2013), Taking Cartography into the Cloud. Kartographische Nachrichten, 63 (4), 191-195.

Peterson, M. (2014), Mapping in the Cloud. Guilford Press, 421 p. 
Regalado, A. (2011), Who Coined 'Cloud Computing'. MIT Technology Review, Business Report, November 2011. http://www.technologyreview.com/news/425970/who-coined-cloud-computing/ (March 30, 2015).

SAMPle, J. \& Ioup, E. (2010), Tile-Based Geospatial Information Systems. Springer, 237 p.

TufTE, E. (1990), Envisioning Information. Graphics Press, 126 p.

VozeniLeK, V. (2015), Aspects of the Thematic Atlas Compilation. In: BRUS, J., VondRAKOVA, A. \& VozenileK, V. (Eds.), Modern Trends in Cartography. Springer, 3-12.

Wolodtschenko, A. (2012), On the new generation of digital mini-atlases. ProceedingsAutoCarto 2012 - Columbus, Ohio, USA - September 16-18, 2012.

http://www.cartogis.org/docs/proceedings/2012/Wolodtschenko_AutoCarto2012.pdf (March 30, 2015).

Yang, C., Goodchild, M., Huang, Q., Nebert, D., Raskin, R., Xu Y., Bambacus, M. \& FAY, D. (2011), Spatial cloud computing: how can the geospatial sciences use and help shape cloud computing? International Journal of Digital Earth, 4 (4), 305-329. 